\title{
Gravitational collapse: A case for thermal relaxation
}

\author{
L. Herrera ${ }^{* \dagger}$ and J. Martínez ${ }^{\ddagger}$
}

\begin{abstract}
Two relativistic models for collapsing spheres at different stages of evolution, which include pre-relaxation processes, are presented. The influence of relaxation time on the outcome of evolution in both cases is exhibited and established. It is shown that relaxation processes can drastically change the final state of the collapsing system. In particular, there are cases in which the value of the relaxation time determines the bounce or the collapse of the sphere.
\end{abstract}

Key words: gravitation - hydrodynamics - stars:mass-loss - stars:neutron

*Área de Física Teórica, Facultad de Ciencias, Universidad de Salamanca, 37008 Salamanca, España.

${ }^{\dagger}$ Permanent address: Departamento de Física, Facultad de Ciencias, Universidad Central de Venezuela, Caracas, Venezuela and Centro de Astrofísica Teórica (C.A.T.), Mérida, Venezuela.

${ }^{\ddagger}$ Grupo de Física Estadística, Departamento de Física, Universidad Autónoma de Barcelona, 08193 Bellaterra, Barcelona, España. e-mail:justino@telemaco.uab.es, phone:+34-3-581 2575 


\section{Introduction}

In the study of gravitational collapse, where dissipative processes play a fundamental role, thermal relaxation time $(\tau)$ is usually neglected. The reason for this may be found in the well known fact that, for most materials at usual laboratory conditions, $\tau$ is very small as compared with typical scales of time of most self-gravitating systems. Thus, it is of the order of $10^{-11} \mathrm{~s}$ for phonon-electron interaction and of the order of $10^{-13} \mathrm{~s}$ for phonon-phonon and free electron interaction at room temperature [1].

There are however situations where relaxation time may not be negligible. Thus, for example, for superfluid Helium, $\tau$ is of the order of $10^{-3} \mathrm{~s}$ for a temperature $1.2 \mathrm{~K}$ [2].

Also, and much more important in the context of the problem considered here, in cores of evolved stars the electron gas is highly degenerate. Then, since the quantum cells of phase space are filled up, such that collisions in which the momentum is changed become rather improbable, the mean free path increase considerably, increasing $\tau$ thereby.

Thus, for example, for a completely degenerate core of radius $\sim 10^{-2} R_{\odot}$ at $T \sim 10^{7} \mathrm{~K}$, the thermal relaxation time is of the order of one second [3]. However, the order of magnitude of relaxation time used here is much lower than that.

Using the expression for the thermal conductivity by electrons in neutron star matter introduced by Flowers and Itho [4, 5], we found (see below) without difficulties relaxation times in the range $\left[10^{-6} \mathrm{~s}, 10^{-4} \mathrm{~s}\right]$.

There have been recent calculations on collapsing systems which include 
pre-relaxation processes [6, 7]. However, either they consider a constant value for the conductivity [6] or they are calculated for a single value of $\tau$ []], excluding the possibility of comparing the evolution for different relaxation times, and thereby, of assessing the influence of $\tau$ on the evolution of the collapsing object.

In this paper we describe the evolution of two different self-gravitating systems dissipating energy through a radial heat flow vector.

Modeling is achieved by using the HJR formalism [8, 9, 10]. However, unlike Di Prisco, Herrera and Esculpi [6] we shall not consider a constant value for thermal conductivity and the astrophysical setting is much more realistic. Also, unlike Martínez [7] we shall follow the approach introduced by Di Prisco et al [6] which allows for comparing the evolution for different values of $\tau$.

The first model is based on the well known Tolman VI solution [11] and may be accomodated to describe to some extent, the core implosion and subsequent bounce, at earlier stages of a supernova explosion.

The second one is more suitable for describing the Kelvin-Helmholtz phase of the birth of a neutron star [12]. However, it is important to emphasize that in spite of the fact that the order of magnitude of different physical variables are well within the range of expected values, our main goal here is not to present a detailed modeling of those scenarios, but to bring out the relevance of pre-relaxation processes in situations when degenerate cores are involved.

The paper is organized as follows. In the next section the field equations and conventions are presented and also a brief resume of the HJR formalism 
is given. The heat conduction equation is presented in section 3, as well as the approach to operate the HJR formalism.

Section 4 is devoted to the description of the two models considered in this paper.

Finally, some conclusions and comments are included in the last section.

Partial differentiation with respect to $u$ and $r$ are indicated by means of the subscripts 0 and 1 respectively. The subscript $a$ denote that the quantity is evaluated at the surface.

\section{The HJR formalism}

We shall consider a non-static distribution of matter which is spherically symmetric, and consists of fluid (which may be locally anisotropic) and heat flow. In Bondi coordinates [13, 15], the metric takes the form

$$
d s^{2}=e^{2 \beta}\left[\frac{V}{r} d u^{2}+2 d u d r\right]-r^{2}\left(d \theta^{2}+\sin ^{2} \theta d \phi^{2}\right)
$$

where $u=x^{0}$ is a time like coordinate $\left(g_{u u}>0\right), r=x^{1}$ is the null coordinate $\left(g_{r r}=0\right)$ and $\theta=x^{2}$ and $\phi=x^{3}$ are the usual angle coordinates. The $u$-coordinate corresponds to the retarded time in flat spacetime and the metric functions $\beta$ and $V$ are functions of $u$ and $r$. A function $\tilde{m}(u, r)$ can be defined by

$$
V=e^{2 \beta}(r-2 \tilde{m}(u, r))
$$

which is the generalization, inside the distribution, of the "mass aspect" defined by Bondi et al [13. In the static limit it coincides with the Schwarzschild 
mass. On the other hand, the radiation coordinates $u, r, \theta$ and $\phi$ can be related to Schwarzschild ones $(T, R, \Theta, \Phi)$ by

$$
\begin{gathered}
T=u+\int_{0}^{r} \frac{r}{V} d r, \\
R=r, \quad \Theta=\theta, \quad \Phi=\phi,
\end{gathered}
$$

and to local Minkowskian coordinates $(t, x, y, z)$ by

$$
\begin{gathered}
d t=e^{\beta}\left(\sqrt{\frac{V}{r}} d u+\sqrt{\frac{r}{V}} d r\right), \\
d x=e^{\beta} \sqrt{\frac{r}{V}} d r \\
d y=r d \theta \\
d z=r \sin \theta d \phi .
\end{gathered}
$$

At the outside of the fluid distribution the space-time is described by the Vaidya metric [14], which in Bondi coordinates is given by $\beta=0$ and $V=r-2 m(u)$.

For the matter distribution considered here, the stress-energy tensor can be written as [7]

$$
T_{\mu \nu}=\left(\rho+P_{\perp}\right) U_{\mu} U_{\nu}-P_{\perp} g_{\mu \nu}+\left(P_{r}-P_{\perp}\right) \chi_{\mu} \chi_{\nu}+2 \mathcal{Q}_{(\mu} U_{\nu)},
$$

where $\rho, P_{r}, P_{\perp}$ are the energy density, radial pressure and tangential pressure respectively as measured by a Minkowskian observer in the Lagrangean frame. The stress-energy tensor outlined above is obtained as measured by an observer using Bondi coordinates. For this observer the four-velocity $U_{\mu}$, and the heat flux vector $\mathcal{Q}^{\mu}$ are given by

$$
U_{\mu}=e^{\beta}\left(\sqrt{\frac{V}{r}} \frac{1}{\left(1-\omega^{2}\right)^{1 / 2}}, \sqrt{\frac{r}{V}}\left(\frac{1-\omega}{1+\omega}\right)^{1 / 2}, 0,0\right),
$$


and

$$
\mathcal{Q}^{\mu}=\mathcal{Q} e^{-\beta}\left(-\sqrt{\frac{r}{V}}\left(\frac{1-\omega}{1+\omega}\right)^{1 / 2}, \sqrt{\frac{V}{r}} \frac{1}{\left(1-\omega^{2}\right)^{1 / 2}}, 0,0\right)
$$

whereas $\chi_{\mu}=-\mathcal{Q}_{\mu} / \mathcal{Q}, \mathcal{Q}=\sqrt{-\mathcal{Q}^{\mu} \mathcal{Q}_{\mu}}$ is the heat flow, and $\omega$ is the velocity of matter as measured by the Minkowski observer defined by (5)-(8).

The Einstein field equations, inside the matter distribution, can be written as [10]:

$$
\begin{gathered}
\frac{1}{4 \pi r(r-2 \tilde{m})}\left(-\tilde{m}_{0} e^{-2 \beta}+(1-2 \tilde{m} / r) \tilde{m}_{1}\right)=\frac{1}{1-\omega^{2}}\left(\rho+2 \omega \mathcal{Q}+P_{r} \omega^{2}\right) \\
\frac{\tilde{m}_{1}}{4 \pi r^{2}}=\frac{1}{1+\omega}\left(\rho-\mathcal{Q}(1-\omega)-P_{r} \omega\right) \\
\beta_{1} \frac{r-2 \tilde{m}}{2 \pi r^{2}}=\frac{1-\omega}{1+\omega}\left(\rho-2 \mathcal{Q}+P_{r}\right), \\
-\frac{\beta_{01} e^{-2 \beta}}{4 \pi}+\frac{1}{8 \pi}\left(1-2 \frac{\tilde{m}}{r}\right)\left(2 \beta_{11}+4 \beta_{1}^{2}-\frac{\beta_{1}}{r}\right)+\frac{3 \beta_{1}\left(1-2 \tilde{m}_{1}\right)-\tilde{m}_{11}}{8 \pi r}=P_{\perp}
\end{gathered}
$$

while outside matter, the stress-energy tensor takes the form

$$
T_{\mu \nu}=-\frac{1}{4 \pi r^{2}} \tilde{m}_{0} \delta_{\mu}^{u} \delta_{\nu}^{u}
$$

and the only non trivial Einstein equation reads

$$
\widetilde{m}_{0}=-4 \pi r^{2} \varepsilon\left(1-\frac{2 \widetilde{m}(u)}{r}\right) .
$$

where $\varepsilon$ is proportional to the energy density of the radiation traveling in the radial direction - see Bondi [15] for details, and subscripts 0,1 denote derivatives with respect to $u$ and $r$ respectively. 
In the HJR formalism [8, 9, 10], one introduces the concept of effective energy density and effective pressure. From (13), the mass function can be expressed as

$$
\tilde{m}=\int_{0}^{r} 4 \pi r^{2} \tilde{\rho} d r
$$

where

$$
\tilde{\rho}=\frac{1}{1+\omega}\left(\rho-\mathcal{Q}(1-\omega)-P_{r} \omega\right),
$$

is the effective energy density, which in the static limit reduces to the energy density of the system.

In a similar way, we can define the effective pressure. From (14) one has

$$
\beta=\int_{a(u)}^{r} \frac{2 \pi r^{2}}{r-2 \tilde{m}} \frac{1-\omega}{1+\omega}\left(\rho-2 \mathcal{Q}+P_{r}\right) d r
$$

or

$$
\beta=\int_{a(u)}^{r} \frac{2 \pi r^{2}}{r-2 \tilde{m}}(\tilde{\rho}+\tilde{P}) d r
$$

with

$$
\tilde{P}=\frac{1}{1+\omega}\left(-\omega \rho-\mathcal{Q}(1-\omega)+P_{r}\right)
$$

being the effective pressure. This one, as the effective energy density, only has a clear physical meaning in the static case, in which it reduces to the radial pressure.

Matching the Vaidya metric to the Bondi metric at the surface $(r=a)$ of the fluid distribution implies $\beta_{a}=\beta(u, r=a)=0$ and the continuity of the mass function $\tilde{m}(u, r)$. The continuity of the second fundamental form must be demanded as well, leading to condition

$$
\dot{a}=-\left(1-2 \frac{\tilde{m}_{a}}{a}\right) \frac{\tilde{P}_{a}}{\tilde{P}_{a}+\tilde{\rho}_{a}} .
$$


- see Herrera and Jiménez [16] for details - where overdot denotes derivative with respect to $u$.

The well-known condition

$$
\mathcal{Q}_{a}=P_{r a},
$$

for radiative spheres [17] can be obtained from the coordinate transformation (5). Effectively, the velocity of matter in Bondi coordinates can be written as

$$
\frac{d r}{d u}=\frac{V}{r} \frac{\omega}{1-\omega},
$$

evaluating the last expression at the surface and comparing it with (23) it follows

$$
\tilde{P}_{a}=-\omega_{a} \tilde{\rho}_{a}
$$

It is easy to show, using (19) and (22), that this condition is equivalent to (24).

The HJR method is based in a system of three surface equations which allows us to find the $u$ dependence of the functions $\beta$ and $\tilde{m}$ present in the field equations (12 15).

To derive the surface equations, usually five dimensionless variables are defined

$$
A \equiv \frac{a}{\widetilde{m}(0)} \quad M \equiv \frac{\widetilde{m}}{\widetilde{m}(0)} \quad u \equiv \frac{u}{\widetilde{m}(0)} \quad F \equiv 1-\frac{2 M}{A} \quad \Omega \equiv \frac{1}{1-\omega_{a}},
$$

where $\widetilde{m}(0)$ is the initial total mass of the system. Then, using (27) and (26), (23) yields the first surface equation. Using the functions just defined into (23) we get the first surface equation

$$
\dot{A}=F(\Omega-1)
$$


which gives the evolution of the radius of the star. From now on, a dot denotes derivative with respect to the dimensionless variable $u / \widetilde{m}(0)$.

The second surface equation can be obtained from the luminosity evaluated at the surface of the system. The luminosity as measured by an observer at rest at infinity reads

$$
L=-\dot{M}=\frac{E}{\left(1+z_{a}\right)^{2}}=E F=\hat{E}(2 \Omega-1) F=4 \pi A^{2} \mathcal{Q}_{a}(2 \Omega-1) F
$$

where $z_{a}$ refers to the boundary gravitational redshift, $\hat{E}$ is the luminosity as seen by a comoving observer, and $E$ is the luminosity measured by a non comoving observer located on the surface. Using relationship (12) and (29) together with the first surface equation we obtain the second one as

$$
\dot{F}=\frac{2 L+F(1-F)(\Omega-1)}{A},
$$

which expresses the evolution of the redshift at the surface.

The third surface equation is model dependent. For anisotropic fluids the relationship $\left(T_{r ; \mu}^{\mu}\right)_{a}=0$ can be written as

$$
\begin{gathered}
\frac{\dot{F}}{F}+\frac{\dot{\Omega}}{\Omega}-\frac{\dot{\tilde{\rho}}_{a}}{\tilde{\rho}_{a}}+F \Omega^{2} \frac{\tilde{R}_{\perp_{a}}}{\tilde{\rho}_{a}}-\frac{2}{A} F \Omega \frac{P_{r a}}{\tilde{\rho}_{a}}= \\
(1-\Omega)\left[4 \pi A \tilde{\rho}_{a} \frac{3 \Omega-1}{\Omega}-\frac{3+F}{2 A}+F \Omega \frac{\tilde{\rho}_{1_{a}}}{\tilde{\rho}_{a}}+\frac{2 F \Omega}{A \tilde{\rho}_{a}}\left(P_{\perp}-P_{r}\right)_{a}\right],
\end{gathered}
$$

being

$$
\tilde{R}_{\perp_{a}}=\tilde{P}_{1_{a}}+\left(\frac{\tilde{P}+\tilde{\rho}}{1-2 \tilde{m} / r}\right)_{a}\left(4 \pi r \tilde{P}+\frac{\tilde{m}}{r^{2}}\right)_{a}-\left(\frac{2}{r}\left(P_{\perp}-P_{r}\right)\right)_{a} .
$$

Expression (31) generalizes the Tolman-Oppenheimer-Volkov equation to the non-static radiative anisotropic case. 
The HJR method [8] allows us to find non static solutions of the Einstein equations from static ones. The algorithm, extended for anisotropic fluids, can be summarized as follows [8, 9]

1. Take a static but otherwise arbitrary interior solution of the Einstein equations for a spherically symmetric fluid distribution

$$
P_{s t}=P(r), \quad \rho_{s t}=\rho(r) .
$$

2. The effective quantities $\tilde{\rho} \equiv \tilde{\rho}(u, r)$ and $\tilde{P} \equiv \tilde{P}(u, r)$ must coincide with $\rho_{s t}$ and $P_{s t}$ respectively in the static limit. We assume that the $r$ dependence in effective quantities is the same that in its corresponding static ones. Nevertheless, note that junction conditions in terms of effective variables, read as (26). This condition allows us to find out the relation between the $u$-dependence of $\tilde{\rho} \equiv \tilde{\rho}(u, r)$ and $\tilde{P} \equiv \tilde{P}(u, r)$. The rationale behind the assumption on the $r$ dependence of the effective variables $\tilde{P}$ and $\tilde{\rho}$, can be grasped in terms of the characteristic times for different processes involved in a collapse scenario. If the hydrostatic time scale $\mathcal{T}_{H Y D R}$, which is of the order $\sim 1 / \sqrt{G \rho}$ (where $G$ is the gravitational constant and $\rho$ denotes the mean density) is much smaller than the Kelvin-Helmholtz time scale $\left(\mathcal{T}_{K H}\right)$, then in a first approximation the inertial terms in the equation of motion can be ignored. Therefore in this first approximation (quasi-stationary approximation) the $r$ dependence of $P$ and $\rho$ are the same as in the static solution. Then the assumption that the effective variables have the same $r$ dependence as the physical variables of the static situation, represents a 
correction to that approximation.

3. Introduce $\tilde{\rho}(u, r)$ and $\tilde{P}(u, r)$, into (18) and (21) to determine $\tilde{m}$ and $\beta$ up to three unknown functions of time.

4. The three surface equations form a system of first order ordinary differential equations, by solving it we find the evolution of the radius, $A(u)$, and two unknown functions of time. These ones can be related with the $u$-dependence of $\tilde{\rho} \equiv \tilde{\rho}(u, r)$ and $\tilde{P} \equiv \tilde{P}(u, r)$.

5. There are four unknown functions of time $(A, F, \Omega$ and $L)$. Thus, it is necessary to impose the evolution of one of them to solve the system of three surface equations. Usually the luminosity is taken as an input function because it can be found from observational data.

6. Once these three functions are known, it is easy to find $\tilde{m}$ and $\beta$. Therefore, the interior metric is completely defined.

7. Now, the left-hand side of the Einstein equations (12)-(15) is known. However, the right-hand side of these equations contain five unknown quantities $\left(\omega, \rho, P_{r}, P_{\perp}\right.$ and $\left.\mathcal{Q}\right)$. Thus, it is necessary to supply another equation to close the system of field equations. In the anisotropic static case the equation of hydrostatic equilibrium reads [18],

$$
P_{\perp}-P_{r}=\frac{r}{2} P_{1}+\left(\frac{\rho+P}{2}\right)\left(\frac{m+4 \pi r^{3} P}{r-2 m}\right) .
$$

This expression is usually generalized, in the context of HJR method, to non-static cases by substituting the physical quantities by the effective 
variables [9, 19]

$$
P_{\perp}-P_{r}=\frac{r}{2} \widetilde{P}_{1}+\left(\frac{\widetilde{\rho}+\widetilde{P}}{2}\right)\left(\frac{\widetilde{m}+4 \pi r^{3} \widetilde{P}}{r-2 \widetilde{m}}\right) .
$$

Now, the Einstein equations, supplemented with (35), form a closed system of equations, and quantities $\omega, \rho, P_{r}, P_{\perp}$ and $\mathcal{Q}$ can be found.

\section{System of Surface Equations: Heat con- duction equation.}

As we mentioned in the previous section, equations (28), (30), and (31) constitute a system of surface equations. However, if we desire to study the influence of the relaxation processes during the collapse, it is necessary to introduce an hyperbolic transport equation. Recently, Di Prisco, Herrera and Esculpi [6] have shown the importance of the relaxation processes involving the heat flow using a Schwarzschild based model with constant thermal conductivity. Our aim is to apply the same procedure to different and more realistic models in order to discern the effects that are model independent and how sensitive is the final state to the presence of relaxation processes.

The system of surface equations can be solved for a given luminosity profile. Thus, the temperature could be found if $\tau$ were known [7]. Nevertheless, this procedure does not give us information about the influence of the relaxation processes on the luminosity profile. Because of this, it seems more convenient to our purposes to follow the method outlined by Di Prisco et al [6]. 
First of all, we assume the evolution of the heat flow governed by the Maxwell-Cattaneo transport equation,

$$
\tau \frac{d \mathcal{Q}^{\nu}}{d s} h_{\nu}^{\mu}+\mathcal{Q}^{\mu}=\chi h^{\mu \nu}\left[T_{, \nu}-T \frac{d U_{\nu}}{d s}\right],
$$

where

$$
\begin{aligned}
& \frac{d \mathcal{Q}^{\nu}}{d s}=U^{\mu} \mathcal{Q}_{; \mu}^{\nu}, \\
& \frac{d U^{\nu}}{d s}=U^{\mu} U_{; \mu}^{\nu},
\end{aligned}
$$

and $\chi$ is the thermal conductivity coefficient. The non vanishing covariant components of the four acceleration read:

$$
\begin{aligned}
\frac{d U_{r}}{d s}=\frac{1}{1+\omega} & {\left[\frac{1}{2 r}-2 \beta_{1}-\frac{1-2 \widetilde{m}_{1}}{2(r-2 \widetilde{m})}\right]+r e^{-2 \beta}\left(\frac{1-\omega}{1+\omega}\right) \frac{\widetilde{m}_{0}}{(r-2 \widetilde{m})^{2}} } \\
- & \frac{1}{(1+\omega)^{2}(1-\omega)}\left[\omega \omega_{1}+r e^{-2 \beta} \frac{1-\omega}{r-2 \widetilde{m}} \omega_{0}\right],
\end{aligned}
$$

and

$$
\frac{d U_{u}}{d s}=e^{-2 \beta}\left(1-\frac{2 \tilde{m}}{r}\right) \frac{\omega}{1-\omega} \frac{d U_{r}}{d s} .
$$

It is easy to demonstrate that in our case, eq. (36) has only one independent component. Thus, evaluating one of them at the surface we obtain

$$
\begin{gathered}
\tau \dot{\mathcal{Q}}_{a}+\mathcal{Q}_{a} \sqrt{F(2 \Omega-1)}= \\
\chi_{a}\left[\dot{T}_{a}-T_{1 a} F(2 \Omega-1)-T_{a} F(2 \Omega-1)\left(\frac{(1-F) \Omega}{2 A F(2 \Omega-1)}+\frac{L}{F^{2} A(2 \Omega-1)}+\frac{\dot{\Omega}}{F(2 \Omega-1)^{2}}\right)\right],
\end{gathered}
$$

where we have used (37), (10), (11), and (11). 
We shall assume that the thermal conduction is dominated by electrons. Thus, the thermal conductivity is given by expression [4, 5]

$$
\chi \simeq 10^{23} \frac{\rho_{14}}{T_{8}} \text { erg. } \mathrm{s}^{-1} \mathrm{~cm}^{-1} \mathrm{~K}^{-1},
$$

where the energy density and the temperature are given in $10^{14} \mathrm{~g} \mathrm{~cm}^{-3}$ and $10^{8} \mathrm{~K}$ units respectively. In the HJR formalism the initial mass is normalized to unity (27). Therefore, all quantities that, in geometrized units, have dimensions of a power of length, are within this framework dimensionless. The expression (40) for the thermal conductivity coefficient can be rewritten as

$$
\chi \simeq \frac{\mathcal{C}}{\xi} \frac{\rho}{T}
$$

being $\mathcal{C}=2.5126 \times 10^{-20}, \rho$ the dimensionless energy density, and $T$ the temperature in Kelvin. The initial mass has been written in terms of the solar mass as $M_{o}=\xi M_{\odot}$, where $\xi$ is a numerical factor.

The energy density in the surface can be written in terms of the heat flow and the effective energy by means of the definition (19). Evaluating it at the surface and using (27) and junction condition (24) we obtain,

$$
\rho_{a}=\mathcal{Q}_{a}+\widetilde{\rho}_{a}\left[\frac{2 \Omega-1}{\Omega}\right] .
$$

Then, evaluating (41) at the surface, the heat transport equation at the surface (39) reads

$$
\begin{gathered}
\tau \dot{\mathcal{Q}}_{a}+\mathcal{Q}_{a} \sqrt{F(2 \Omega-1)}=\frac{\mathcal{C}}{\xi}\left(\mathcal{Q}_{a}+\widetilde{\rho}_{a}\left[\frac{2 \Omega-1}{\Omega}\right]\right) \times \\
{\left[\frac{\dot{T}_{a}}{T_{a}}-\frac{T_{1 a}}{T_{a}} F(2 \Omega-1)-F(2 \Omega-1)\left(\left(\frac{1-F}{2 A F}\right) \frac{\Omega}{2 \Omega-1}+\frac{L}{F^{2} A(2 \Omega-1)}+\frac{\dot{\Omega}}{F(2 \Omega-1)^{2}}\right)\right] .}
\end{gathered}
$$




\subsection{Boundary condition for the transport equation}

Expression (43) relates the heat flow and the temperature at the surface to the temperature gradient through quantities present in the surface equations (28), (30), and (31).

The connection, at the surface, between the heat flow and the temperature can be found by means of the effective temperature $T_{\text {eff }}$. This one is defined by means of the luminosity perceived by an observer located momentarily on the surface,

$$
E=\left[4 \pi r^{2} \sigma T_{e f f}^{4}\right]_{r=a}
$$

being $\sigma$ the Steffan-Boltzmann radiation constant. Thus, the effective temperature would be the temperature at the surface of the star if it would radiate as a black body. This concept is linked up with the theory of stellar atmospheres by means of the photosphere [20, p. 70], [21, p. 586] and [22, p. 295]. The photosphere comprises the most external layers of the star, and its thickness is determined by the optical depth for photons. At the surface this optical depth vanishes and the effective temperature is related to the material one by the expression

$$
T_{a}^{4}=\frac{1}{2} T_{e f f}^{4}
$$

Then, by substitution of (45) and (44) into (29) we obtain

$$
\mathcal{Q}_{a}=T_{a}^{4} \frac{2 \sigma}{2 \Omega-1} .
$$

Introducing this expression in (43), we obtain the fourth surface equation

$$
\dot{y}\left[\frac{2 y^{4} \xi^{2}}{2 \Omega-1}\left(4 \tau-\frac{\mathcal{C}}{\xi}\right)-\widetilde{\rho}_{a} \frac{\mathcal{C}}{\xi}\left(\frac{2 \Omega-1}{\Omega}\right)\right]=\xi^{2} y^{5}\left[\frac{4 \tau \dot{\Omega}}{(2 \Omega-1)^{2}}-\frac{2 \sqrt{F}}{\sqrt{2 \Omega-1}}\right]-
$$




$$
\frac{\mathcal{C}}{\xi}\left[\frac{2 y^{4} \xi^{2}}{2 \Omega-1}+\widetilde{\rho}_{a}\left(\frac{2 \Omega-1}{\Omega}\right)\right]\left(y_{1} F(2 \Omega-1)+y \Phi\right),
$$

where

$$
\Phi=\left(\frac{1-F}{2 A}\right) \Omega+8 \pi A y^{4} \xi^{2}+\frac{\dot{\Omega}}{2 \Omega-1} .
$$

the function $y$ has been defined as

$$
y^{4} \equiv \zeta T_{a}^{4}
$$

and the constant $\zeta \equiv \sigma M_{\odot}^{2} \simeq 3.4097 \times 10^{-54} \mathrm{~K}^{-4}$ is calculated taken $M_{\odot}$ in geometrized units. The luminosity $L$, present in (43), can be written in terms of $y$ by means of (29), (45) and (44)

$$
L=8 \pi A^{2} y^{4} \xi^{2} F
$$

Note that all terms in (47) are dimensionless, including the relaxation time $\tau$, which is related to the physical $\tau\left(\equiv \tau_{p h}\right)$ by means of

$$
\tau=\tau_{p h} \frac{c^{3}}{G m_{o}} \simeq 2.0298 \times 10^{5} \frac{\tau_{p h}}{\xi} \mathrm{s}^{-1} .
$$

where $c$ and $G$ denotes the speed of light and the gravitational constant, and $m_{o}$ is the total mass in grams.

\subsection{The DHE approach.}

As we mentioned above we shall use the approach introduced by Di Prisco et al [6] (DHE approach henceforth) to solve the system of surface equations. This system of equations usually is composed of three differential equations which give the evolution of three quantities: The radius (28), the boundary gravitational redshift (30) and the collapse velocity of the surface (31). In 
order to study the effect of the thermal relaxation processes on the system it is necessary to include an additional equation accounting for the evolution of the temperature at the surface. Thus, expression (47) constitutes the fourth surface equation. The method can be described as follows:

1. Solve, as usual, the system of surface equations (28), (30), and (31) for a given luminosity $L$.

2. Introduce the results into the fourth surface equation (47), and solve if for $\tau=0$ (Eckart's law). Since $L$ is known, equation (50) allows us to find the temperature evolution and equation (47) provides us the temperature gradient.

3. Use the evolution found for $T_{1 a}$ in the resolution of the complete system of surface equations (28), (30), (31), and (47) for non vanishing $\tau$ (Maxwell-Cattaneo law). So that the evolution of $A, F$ and $L$ is found for a given value of $\tau$ and we are able to compare it with that found for $\tau=0$.

The method described above must be self consistent, i.e. we must recover the luminosity profile assumed in the first point if a vanishing relaxation time is imposed in the third one.

The procedure adopted here assumes that the temperature gradient is independent of the adopted value for $\tau$. This hypothesis can be justified in the following terms: The existence of a temperature gradient is caused by local processes. As a consequence of its presence a non-vanishing flux appears, giving rise to dissipative processes. The relation between cause and 
effect is, in this case, non linear due to the fact that dissipative processes can change the local situation. On the other hand, relaxation processes act directly on dissipative processes. Thus, they must affect in a indirectly way to the local situation. Nevertheless, the effect on local processes must be less important than on dissipative ones having, then, a little influence on temperature gradients. In first approximation we assume that, for time-scales of the order of $\tau$, the influence of the relaxation processes on temperature gradients can be neglected.

In order to ascertain the goodness of this assumption, we have numerically integrated the system of surface equations (28), (30), (31) and (47), for the models presented below, assuming the same luminosity profile for a wide range of values of $\tau$. The differences found among temperature gradients for different $\tau$ can be neglected. This fact reinforces the adopted hypothesis. The unsensibility of the inner temperature to the relaxation time for a fixed luminosity [7] points in the same direction as well.

\section{The models}

The HJR method starts from a static solution of the Einstein equations (sec. II). We shall study the evolution of two different models. The first one, corresponds to the well-known Tolman VI solution [11], while the second one comes from a static solution due to Gokhroo and Mehra 23. 


\subsection{Tolman VI-type solution}

The equation of state obtained from the Tolman VI static solution approaches, in the core of the star, to a highly compressed Fermi gas. The energy density and radial pressure are

$$
\rho(r)_{s t}=\frac{3}{56 \pi r^{2}},
$$

and

$$
P(r)_{s t}=\frac{\rho}{3}\left[\frac{9 a-9 r}{9 a-r}\right]
$$

respectively. In order to generate non static solutions, Tolman VI model has been used often in the HJR framework as a departing solution [8]. Following the HJR method, the effective quantities are taken as

$$
\widetilde{\rho}=\frac{3 G(u)}{r^{2}},
$$

and

$$
\widetilde{P}=\frac{\widetilde{\rho}}{3}\left[\frac{1-9 K(u) r}{1-K(u) r}\right] .
$$

Using expressions (54), (18) and (27) the function $G(u)$ reads,

$$
G=\frac{1-F}{24 \pi} .
$$

Function $K(u)$ follows, as a function of $\Omega$, from junction condition (26), (54), (55) and (27),

$$
K=\frac{1}{3 A}\left[\frac{3-4 \Omega}{1-4 \Omega}\right] .
$$

By means of the last four expressions, the system of surface equations (28), (30), (31), and (47) reads

$$
\dot{A}=F(\Omega-1),
$$




$$
\begin{gathered}
\dot{F}=\frac{2 L+F(1-F)(\Omega-1)}{A}, \\
\frac{\dot{F}}{F}+(1-F) \frac{\dot{\Omega}}{\Omega}=\frac{F(1-F)(4 \Omega-3)(4 \Omega-1)}{8 A}-\frac{(1-F)^{2}}{2 A \Omega}+\frac{4 L \Omega}{(2 \Omega-1) A},
\end{gathered}
$$

and

$$
\begin{gathered}
\dot{y}\left[\frac{2 y^{4} \xi^{2}}{2 \Omega-1}\left(4 \tau-\frac{\mathcal{C}}{\xi}\right)-\frac{\mathcal{C}}{\xi}\left(\frac{1-F}{8 \pi A^{2}}\right)\left(\frac{2 \Omega-1}{\Omega}\right)\right]=\xi^{2} y^{5}\left[\frac{4 \tau \dot{\Omega}}{(2 \Omega-1)^{2}}-\frac{2 \sqrt{F}}{\sqrt{2 \Omega-1}}\right]- \\
\frac{\mathcal{C}}{\xi}\left[\frac{2 y^{4} \xi^{2}}{2 \Omega-1}+\left(\frac{1-F}{8 \pi A^{2}}\right)\left(\frac{2 \Omega-1}{\Omega}\right)\right]\left(y_{1} F(2 \Omega-1)+y \Phi\right),
\end{gathered}
$$

This model can be applied in the context of the bouncing of the core during a supernova explosion. It is important to note that the value of the quantity $M / A$ plays a fundamental role in the evolution of the system. In particular, for the model under consideration the bounce of the surface occurs if $M / A \leq 3 / 14$ [8]. As Di Prisco et al [6] showed, this quantity is influenced by the inclusion of relaxation processes. If this effect is model independent then, in some cases, it will cause a drastic change in the final state of the collapsing core.

In order to evaluate the influence of non vanishing $\tau$ in the evolution of the system, we assume as initial configuration:

$$
A_{o}=6, \Omega_{o}=0.878497, F=\frac{2}{3}, \text { and } M_{o}=1.3 M_{\odot} .
$$

This corresponds to a core with an energy density about $10^{14} \mathrm{~g} \mathrm{~cm}^{-3}$ in the external layers, whereas its radius is $11521 \mathrm{~m}$.

In the case of vanishing relaxation time, the luminosity is taken as

$$
L=\frac{2 M_{r}}{\sqrt{x^{3} \pi}} \sqrt{t} e^{-t / x}
$$


where $M_{r}$ is the total radiated mass, and $x$ is an arbitrary parameter which determines the decaying time of $L$. In this case $x$ has been taken as 10 and $M_{r}=8.407 \times 10^{-6}$. This brings a maximum luminosity around $1.5 \times 10^{53}$ $\mathrm{erg} \mathrm{s}^{-1}$, and a characteristic time for $L$ close to $0.5 \mathrm{~ms}$. The total radiated energy is of the order of $10^{49} \mathrm{erg}$.

Following the DHE method described in the previous section, the system of surface equations (58)-(61) can be solved for $\tau=0$ using (63). This allows us to find the temperature gradient in the surface, which is used to solve (58)-(61) for $\tau \neq 0$.

The luminosity profile is displayed in fig. 1 for several values of $\tau$. The maximum luminosity decreases if $\tau$ grows. Thus, the higher $\tau$, the softer collapse. Other effect that can be inferred from figure 1 refers to the width of the pulse of luminosity. This one is larger for larger $\tau$ 's. The same influence can be observed in the temperature of the surface (fig. 2). Note that the maximum luminosity and temperature takes place at larger times as $\tau$ increases. This is due, as corresponds to a relaxation process, to the fact that for larger values of the relaxation time, the system requires more time to establish the heat flow.

As we mentioned above, the quantity $M / A$ is greatly influenced by the presence of relaxation processes (figure 3). An interesting effect must be noted in its evolution. The $M / A$ profile presents a sort of bifurcation for $\tau=\tau_{\text {bif }} \sim 3.5 \times 10^{-6}$ s. If $\tau>\tau_{\text {bif }}$, the core evolves towards a more compact final state than the initial one. The situation is reversed for $\tau<\tau_{b i f}$. Thus, small values of $\tau$ have a great influence in the final state. Something similar occurs in the evolution of the radius (figure 4). The bounce of the surface 
takes place if $\tau<\tau_{b i f}$, whereas for $\tau>\tau_{b i f}$ the collapse is ensured. This fact can be explained taking into account the influence of the luminosity in the evolution of the collapse. In the present model, and for $\tau=0$, the pulse of luminosity is the responsible of the bounce of the surface. In case when luminosity is low, the explosion won't take place. The presence of relaxation processes imposes a lower luminosity. Therefore, for $\tau>\tau_{b i f}$ the luminosity will be insufficient to stop the collapse, and the surface won't bounce. There are cases in which, as in the studied model, small variations in the luminosity make the bounce impossible.

The influence of thermal relaxation processes seems to be important in the evolution of the collapsing system. Nevertheless, the question is if the value found for $\tau_{b i f}$ is close enough to the expected value for the relaxation time. Assuming that the thermal conduction is dominated by electrons, the relaxation time can be roughly estimated, in the limit of high frequencies, by the expression [6]

$$
\tau \sim \frac{10^{20}}{T^{2} v^{2}} \mathrm{~s}
$$

where $T$ is measured in $\mathrm{K}$ and thermal signals travel with speed $v$ given in $\mathrm{cm}$ $\mathrm{s}^{-1}$. This expression is only valid assuming $\rho$ and $\chi$ as constants. However, it can be taken as an indicative of the order of magnitude for $\tau$. Assuming $T \sim 10^{10} \mathrm{~K}$, and that thermal signals propagating approximately at $10^{3} \mathrm{~cm}$ $\mathrm{s}^{-1}$ (the second sound speed in superfluid helium) we obtain $\tau \sim 10^{-6} \mathrm{~s}$. Thus, the obtained $\tau_{b i f}$ must be considered as a realistic value for $\tau$ and the effects described above take a special importance.

The total radiated mass, as a function of $\tau$, for two different initial and 
boundary conditions is presented in figure 5. One of them (TOL06 henceforth) corresponds to initial conditions (62) and boundary condition (63). The other (TOL04 from now on) has been solved using

$$
A_{o}=6.5, \Omega_{o}=0.845215, F=\frac{9}{13}, \text { and } M_{o}=1.3 M_{\odot},
$$

as initial conditions, and assuming a Gaussian pulse profile for the luminosity

for vanishing $\tau$. The total radiated mass, if $\tau=0$, is $M_{r}=10^{-4} M_{o}$, and the maximum luminosity is about $7.2 \times 10^{54} \mathrm{erg} \mathrm{s}^{-1}$. The point marked A in figure 5 indicates the limit of validity for TOL04 model. The Tolman VI solution fails for large times. In particular, TOL04 model fails before the luminosity may vanish if $\tau$ is larger than $\sim 0.07 \mathrm{~ms}$. TOL04 model is interesting because it clearly shows the non linearity, between the total radiated mass, and $\tau$. This effect occurs in TOL06 model as well, but is only visible for very small relaxation times and the effect is not so accused. In spite of the maximum luminosity decreasing as $\tau$ increases, the total time in which the system radiates grows with the relaxation time, and the total radiated mass too. Nevertheless, the maximum value of the total radiated mass is bounded. This upper limit to $M_{r}$ is reached if relaxation time is of the order of the width of the luminosity profile.

\subsection{Gokhroo \& Mehra-type solution}

This solution corresponds to an anisotropic fluid with variable energy density. The choice of this solution is based on the fact, that it leads to densities and pressures similar to the well-known Bethe-Börner-Sato (BBS) Newtonian 
state equation [21, 24, 25]. Its generalization to the HJR method has been done recently with good results [7].

In this solution the energy density and radial pressure are assumed as 23]

$$
\rho(r)_{s t}=\rho_{c}\left(1-k \frac{r^{2}}{a^{2}}\right)
$$

and

$$
P_{r}(r)_{s t}=P_{c}\left(1-\frac{2 m}{r}\right)\left(1-\frac{r^{2}}{a^{2}}\right)^{n},
$$

where the constants $k$ and $n$ are within the range $0 \leq k \leq 1$ and $n \geq 1$. The central energy density $\rho_{c}$, and central radial pressure $P_{c}$ are related by expression

$$
P_{c}=\lambda \rho_{c}
$$

The tangential pressure is given by

$$
\begin{gathered}
P_{\perp}(r)-P_{r}(r)=\frac{r}{2}\left[P_{r}\right]_{1}+\left(\frac{\rho+P_{r}}{2}\right)\left(\frac{m(r)+4 \pi r^{3} P_{r}}{r-2 m(r)}\right) \\
=\frac{3}{10} \frac{k P_{c}}{a^{2}} \alpha r^{4}\left(1-\frac{r^{2}}{a^{2}}\right)^{n}+\frac{r^{2}}{2\left(1-\frac{2 m}{r}\right)} \Phi,
\end{gathered}
$$

where

$$
\begin{gathered}
\Phi=2 P_{c}\left(1-2 \frac{m}{r}\right)^{2}\left[2 \pi P_{c}\left(1-\frac{r^{2}}{a^{2}}\right)^{2 n}-\frac{n}{a^{2}}\left(1-\frac{r^{2}}{a^{2}}\right)^{n-1}\right] \\
+\frac{\alpha \rho_{c}}{2}\left(1-\frac{3 k}{5} \frac{r^{2}}{a^{2}}\right)\left(1-k \frac{r^{2}}{a^{2}}\right)
\end{gathered}
$$

being

$$
\alpha=\frac{8 \pi \rho_{c}}{3}
$$


The effective quantities are defined, following the second point of the HJR method, as - see Martínez [7] for details -

$$
\widetilde{\rho}=\widetilde{\rho}_{c}(u)\left(1-K(u) \frac{r^{2}}{a^{2}}\right),
$$

and

$$
\widetilde{P}=\widetilde{P}_{c}(u)\left(1-2 \frac{\widetilde{m}}{r}\right)\left(1-G(u) \frac{r^{2}}{a^{2}}\right)^{n} .
$$

Where the functions $\widetilde{\rho}_{c}(u)$ and $\widetilde{P}_{c}(u)$, are defined by

$$
\widetilde{\rho}_{c}(u)=\rho_{c} \frac{K(u)}{K(0)} \equiv \rho_{c} \frac{K}{K_{o}},
$$

and

$$
\widetilde{P}_{c}(u)=P_{c} \frac{K(u)}{K(0)} \equiv P_{c} \frac{K}{K_{o}} .
$$

The functions of time $K(u)$, and $G(u)$ are found to be

$$
K(u)=\left\{\begin{array}{ll}
\frac{5}{6}\left[1+\sqrt{\left.1-\left(\frac{12 K_{o}}{5 \alpha}\right)\left(\frac{1-F}{A^{2}}\right)\right]}\right. & \text { if } K_{o}>\frac{5}{6} \\
\frac{5}{6}\left[1-\sqrt{\left.1-\left(\frac{12 K_{o}}{5 \alpha}\right)\left(\frac{1-F}{A^{2}}\right)\right]}\right. & \text { if } K_{o}<\frac{5}{6}
\end{array},\right.
$$

and

$$
G(u)=1-\left[\frac{(1-\Omega)(1-K)}{F \Omega \lambda}\right]^{1 / n} .
$$

The expression for tangential pressure can be found following the point seven of the HJR method.

Thus, the system of surface equations for this model is

$$
\begin{gathered}
\dot{A}=F(\Omega-1), \\
\dot{F}=\frac{1}{A}[2 L+F(1-F)(\Omega-1)],
\end{gathered}
$$




$$
\dot{\Omega}=-\frac{\dot{F}}{F} \Omega+\frac{\dot{K}}{K} \frac{(1-2 K)}{(1-K)} \Omega+\frac{4 L \Omega^{2}}{3 \widetilde{\alpha} A^{3}(2 \Omega-1)(1-K)}+\Omega(1-\Omega) \Lambda,
$$

and

$$
\begin{gathered}
\dot{y}\left[\frac{2 y^{4} \xi^{2}}{2 \Omega-1}\left(4 \tau-\frac{\mathcal{C}}{\xi}\right)-\rho_{c} \frac{\mathcal{C}}{\xi}\left(\frac{K(1-K)}{K_{o}}\right)\left(\frac{2 \Omega-1}{\Omega}\right)\right]=\xi^{2} y^{5}\left[\frac{4 \tau \dot{\Omega}}{(2 \Omega-1)^{2}}-\frac{2 \sqrt{F}}{\sqrt{2 \Omega-1}}\right]- \\
\frac{\mathcal{C}}{\xi}\left[\frac{2 y^{4} \xi^{2}}{2 \Omega-1}+\rho_{c} \frac{K(1-K)}{K_{o}}\left(\frac{2 \Omega-1}{\Omega}\right)\right]\left(y_{1} F(2 \Omega-1)+y \Phi\right),
\end{gathered}
$$

where

$$
\begin{gathered}
\Lambda=\frac{3 \alpha K}{2 K_{o}} A(1-K)\left(\frac{3 \Omega-1}{\Omega}\right)-\frac{3+F}{2 A}+\frac{2 F \Omega}{A(1-K)}(\Psi-K) \\
\Psi=\frac{3}{10 K_{o}} \lambda \alpha A^{2} K^{2}(1-G)^{n} \\
+\frac{A^{2}}{2 F}\left[\frac{3 \alpha K}{2 K_{o}} \lambda^{2} F^{2}(1-G)^{2 n}-\frac{2 n \lambda G}{A^{2}} F^{2}(1-G)^{n-1}+\frac{\alpha}{2}\left(1-\frac{3 K}{5}\right) \frac{K(1-K)}{K_{o}}\right],
\end{gathered}
$$

and $\Phi$ is defined in (48).

We have integrated the system of surface equations for this model with the initial data $A(0)=10 ; \Omega(0)=1$, and with $n=1 ; \lambda=1 / 3 ; K(0)=0.999$. Also, the inital mass is $1.3 M_{\odot}$. This corresponds to a star with an initial radius of 19.201 meters, a central density equal to $2.17 \times 10^{14} \mathrm{gcm}^{-3}$ and a surface density equal to $2.17 \times 10^{11} \mathrm{~g} \mathrm{~cm}^{-3}$. For the luminosity we have taken a gaussian pulse with center at $u=200$ and width equal to 20 (in dimenssionless $u$-units), which is equivalent to a width of $0.13 \mathrm{~ms}$.

In the case $\tau=0$, the collapse spans over $u \sim 400(2.5 \mathrm{~ms})$. As $\tau$ increases, that time also increases and may be as large as $32 \mathrm{~ms}$ for $\tau=900(5.7 \mathrm{~ms})$.

In figure 6 we plot the ratio of the total radiated mass divided by the total radiated mass for $\tau=0$, as function of $\tau$, for different radiated mass in the $\tau=0$ case. 
The sensitivity of this ratio to different values of $\tau$ is clearly exhibited. This fact is also present in the Tolman VI model discussed in the previous section (fig.5). However in this model non linear effects seem to be stronger than in the precedent one and the ratio may be larger or smaller than one, depending on the total radiated mass.

The evolution of the radius is exhibited in figures 7 and 8 for different total radiated mass ( $1 \%$ and $0.001 \%$ of the total mass) and different $\tau$ 's. The dependence of the final value of the radius on $\tau$, is linear for low emission $(0.001 \%)$ whereas it is not for the stronger case $(1 \%)$.

The ratio $M_{\text {final }} / A_{\text {final }}$ behaves essentially as the other surface variables (fig.9). The resulting object is more compact for larger luminosities, however as $\tau$ increases, the final configuration is less compact and it may happen that for sufficiently large $\tau$ 's, the object may be at the end of it's evolution less compact than in the $\tau=0$ case.

Finally, figures 10 and 11 show the evolution of luminosity profiles for different radiated mass and different $\tau$ 's. As in all known models, larger $\tau$ 's means longer emissions and more flattened pulses. This effect is sharper for stronger emissions.

\section{Conclusions}

It has been the purpose of this paper to exhibit the relevance of thermal relaxation time in the problem of collapse, by means of two models of radiating spheres. It is important to emphasize that this relaxation time is systematically neglected in collapse calculations, where processes may occur 
on time scales which may be of the order of magnitude of (or at least not much larger than) relaxation time, leading thereby to incorrect conclusions.

One of the models (Tolman VI) is suitable for describing the evolution of the core at the earliest stages of a supernova explosion.

The second one (G-M) is more adapted to describe the Kelvin-Helmoltz phase in the birth of a neutron star.

However, due to time restrictions in numerical calculation, in this model (G-M), we have integrated the surface equation over a period of about tens of milliseconds, instead of tens of seconds, which is the typical time of the Kelvin-Helmholtz phase in neutron star formation. Consequently all times have to be scaled by the same factor.

Preliminary results show that in what the relaxation time concerns, its influence on the evolution of the object is not qualitatively changed by this "scaling".

In the Tolman VI model, the point to emphasize is the bifurcation introduced by changes in $\tau$ of the order $10^{-6} \mathrm{~s}$. Such values are within the range of possible values of $\tau$, and therefore the study of the evolution of such system seems to require a good account of pre-relaxation processes.

In the second model the dependence of the final configuration on $\tau$ may be non-linear and is affected by the total emission.

At any rate the final configuration is clearly $\tau$-dependent.

It is worth mentioning that the hydrostatic time scale for the second model is of the order of $0.29 \mathrm{~ms}$, which clearly indicates that in these calculations the hydrostatic approximation is not a very good one. This is an additional argument to use the HJR-formalism, which in some sense may be 
envisaged as a "correction" to hydrostatic approximation (see point 2 of the algorithm in section 2).

We would like to conclude with the following comment: In a collapse calculation, neutrino transport plays an important role, the reason to overlook this issue here, resides in the fact that we are not concerned with the problem of modeling gravitational collapse, but with the influence of thermal relaxation time in the outcome of evolution. Conduction associated with trapped neutrinos is certainly of greatest relevance, and it should be very interesting to find out the role played by relaxation time of that process. This however is out of the scope of this paper.

\section{Acknowledgments}

This work was partially supported by the Spanish Ministry of Education under Grant No. PB94-0718 


\section{References}

[1] Peirls R., 1956, Quantum Theory of Solids, Oxford University Press, London.

[2] Band W., Meyer L., 1948, Phys. Rev., 73, 226.

[3] Harwit M., 1988, Astrophysical Concepts, Springer-Verlag, New York.

[4] Flowers N., Itoh N., 1979, ApJ, 230, 847

[5] Flowers N., Itoh N., 1979, ApJ, 250, 750

[6] Di Prisco A., Herrera L., Esculpi M., 1996, Class. Quantum Gravit., 13, 1053

[7] Martínez J., 1996, Phys. Rev. D, 53, 6921

[8] Herrera L., Jiménez J., Ruggeri G.J., 1980, Phys. Rev. D., 22, 2305

[9] Cosenza M., Herrera L., Esculpi M., Witten L., 1982, Phys. Rev. D, 25, 2527

[10] Herrera L., Jiménez J., Esculpi M., 1987, Phys. Rev. D., 36, 2986

[11] Tolman R., 1939, Phys. Rev., 55, 364

[12] Burrows A., Lattimer L., 1986, ApJ.,307, 178

[13] Bondi H., van der Burg M.G.J., Metzner A.W.K., 1962, Proc. R. Soc. London, A269, 21 
[14] Vaidya P.C., 1951, Proc. Ind. Acad. Sci. Sect. A, 33, 264

[15] Bondi H., 1964, Proc. R. Soc. London, A281, 39

[16] Herrera L., Jiménez J., 1983, Phys. Rev. D, 28, 2987

[17] Santos N.O., 1985, MNRAS, 216, 403

[18] Cosenza M, Herrera L., Esculpi M., Witten L., 1981, J. Math. Phys., 22,118

[19] Barreto W., Rojas S., 1992, Ap\&SS, 193, 201

[20] Kippenhan R., Weigert A., 1994, Stellar Structure and Evolution. 3th printing, Springer Verlag, Berlin

[21] Shapiro S.L., Teukolsky S.A., 1983, Black Holes, White Dwarfs and Neutron Stars. John Wiley \& Sons, New York

[22] Hansen C.J., Kawaler S.D., 1994, Stellar Interiors Physical Principles, Structure, and Evolution. Springer, New York

[23] Gokhroo M.K., Mehra A.L., 1994, Gen. Rel. Grav., 26, 75

[24] Börner G., 1973, On the properties of Matter in Neutron Stars. Springer Tracts in Physics, Springer, Berlin

[25] Demiański M., 1985, Relativistic Astrophysics. International Series in Nat. Phyl. V110, Ed. D. Ter Haar, Pergamon Press Oxford 


\section{Figure captions}

Figure 1.- Luminosity profile as a function of time. In all figures the values of the relaxation time are given in miliseconds.

Figure 2.- Evolution of the temperature in the surface for different values of $\tau$.

Figure 3.- Evolution of $M / A$ for different values of $\tau$. Note the bifurcation between $\tau=0.0032 \mathrm{~ms}$ and $\tau=0.0038 \mathrm{~ms}$.

Figure 4.- Radius as a function of time. The bounce is only possible for $\tau<0.0035 \mathrm{~ms}$.

Figure 5.- Ratio between total radiated mass and total radiated mass for $\tau=0$ as a funtion of the relaxation time. Dashed and solid lines correspond to TOL04 and TOL06 models respectively. TOL04 model fails for $\tau$ larger than the corresponding to point marked A.

Figure 6.- Same as fig. 5 for G-M model. Curves are labeled with the percentage of radiated mass in the case $\tau=0$.

Figure 7.- Variation in the radius for $M_{r}=10^{-5}(0.001 \%)$

Figure 8.- Same as fig. 7 for $M_{r}=0.01$ (1\%). Note that in the low emission case (fig. 7) the variation is given in $\mathrm{cm}$, while in this case $\Delta A$ is given in meters.

Figure 9.- Ratio between final value of $M / A$ and $M / A$ for $\tau=0$ as a function of $\tau$. The labels in each curve means the same as in fig. 6 . 
Figure 10.- Luminosity profile for different values of $\tau$. The radiated mass in the case $\tau=0$ is a $0.001 \%$ of the initial mass.

Figure 11.- Same as fig. 10. The radiated mass for $\tau=0$ is a $1 \%$ of the initial mass. 


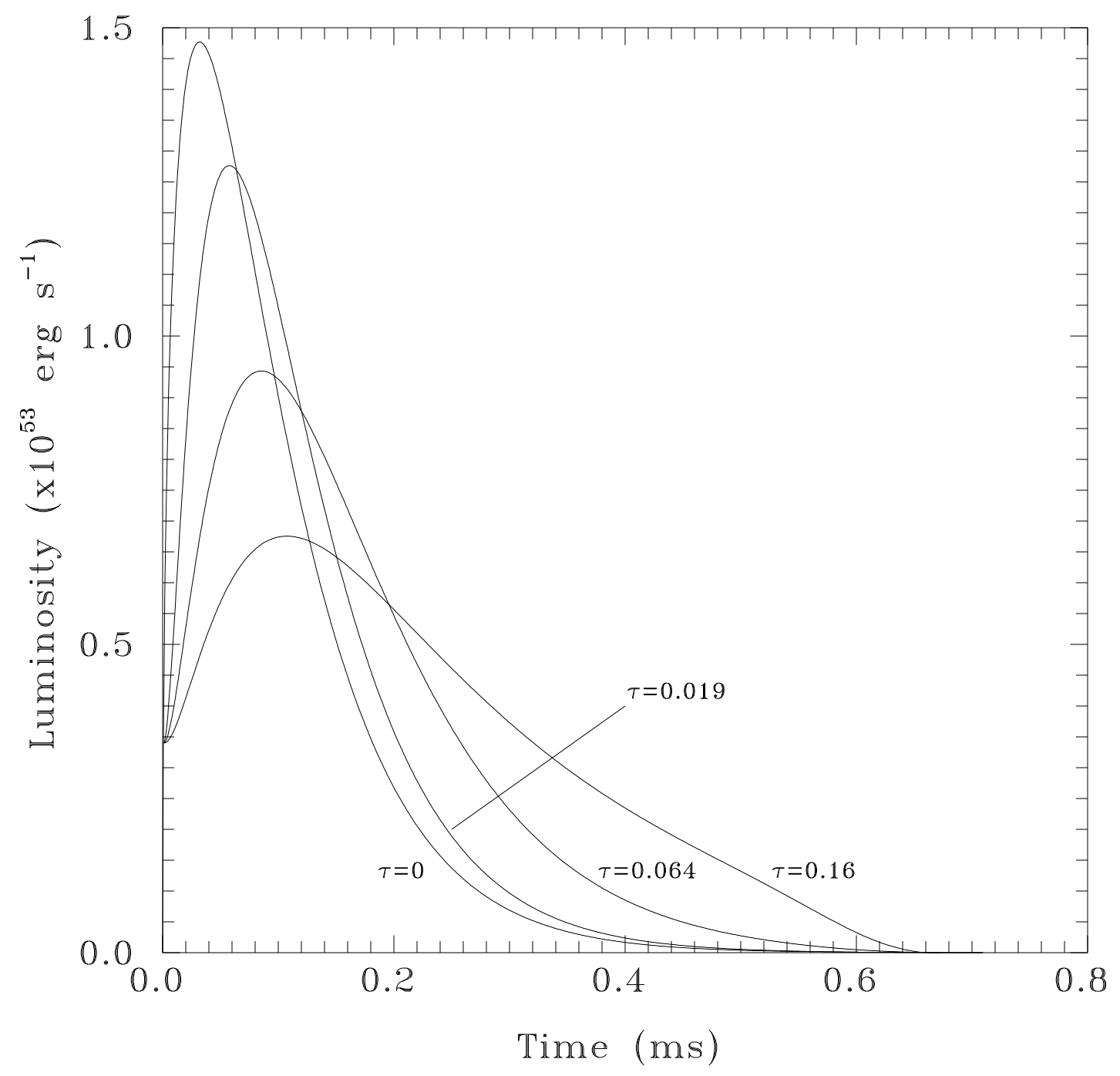




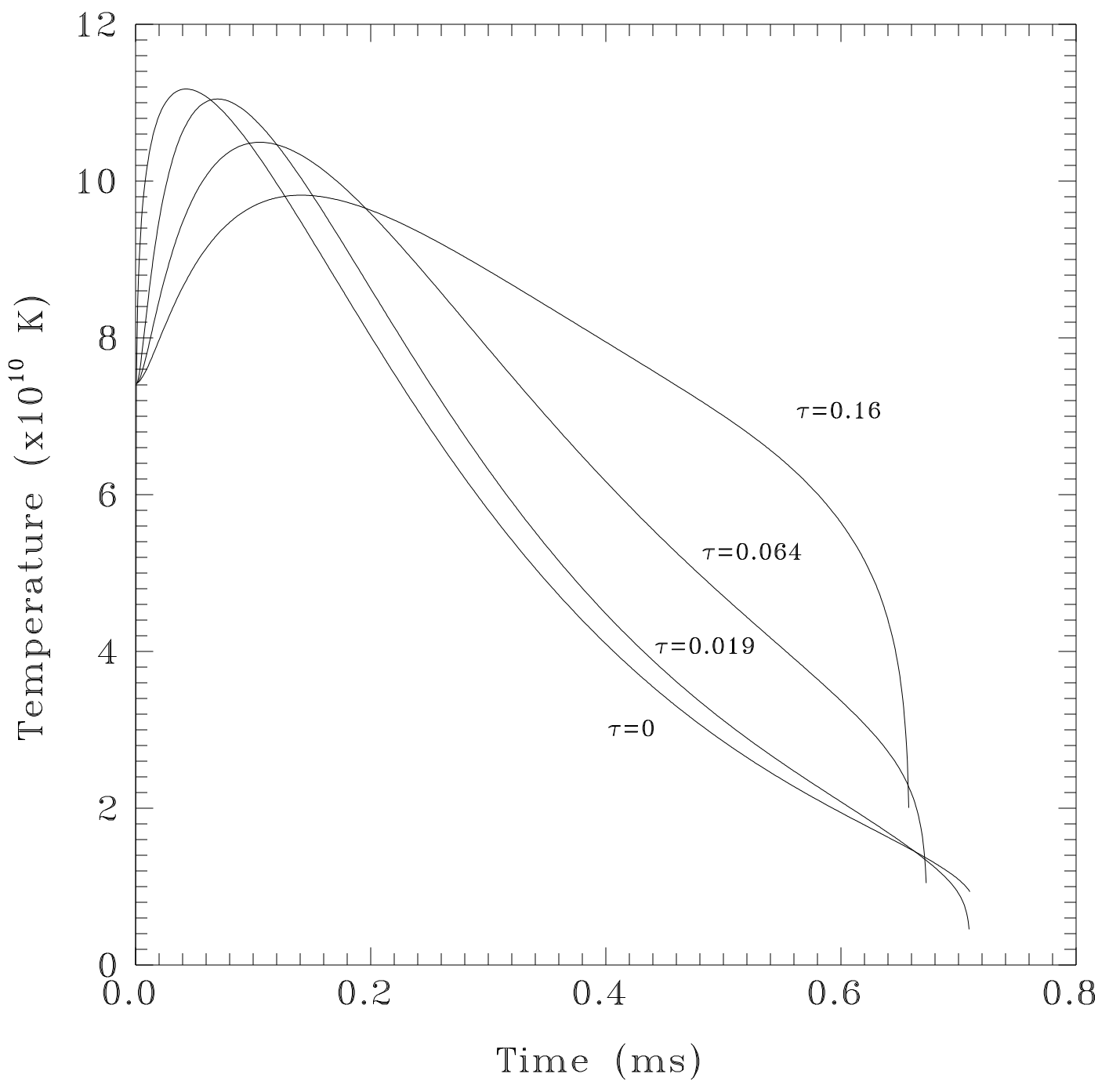




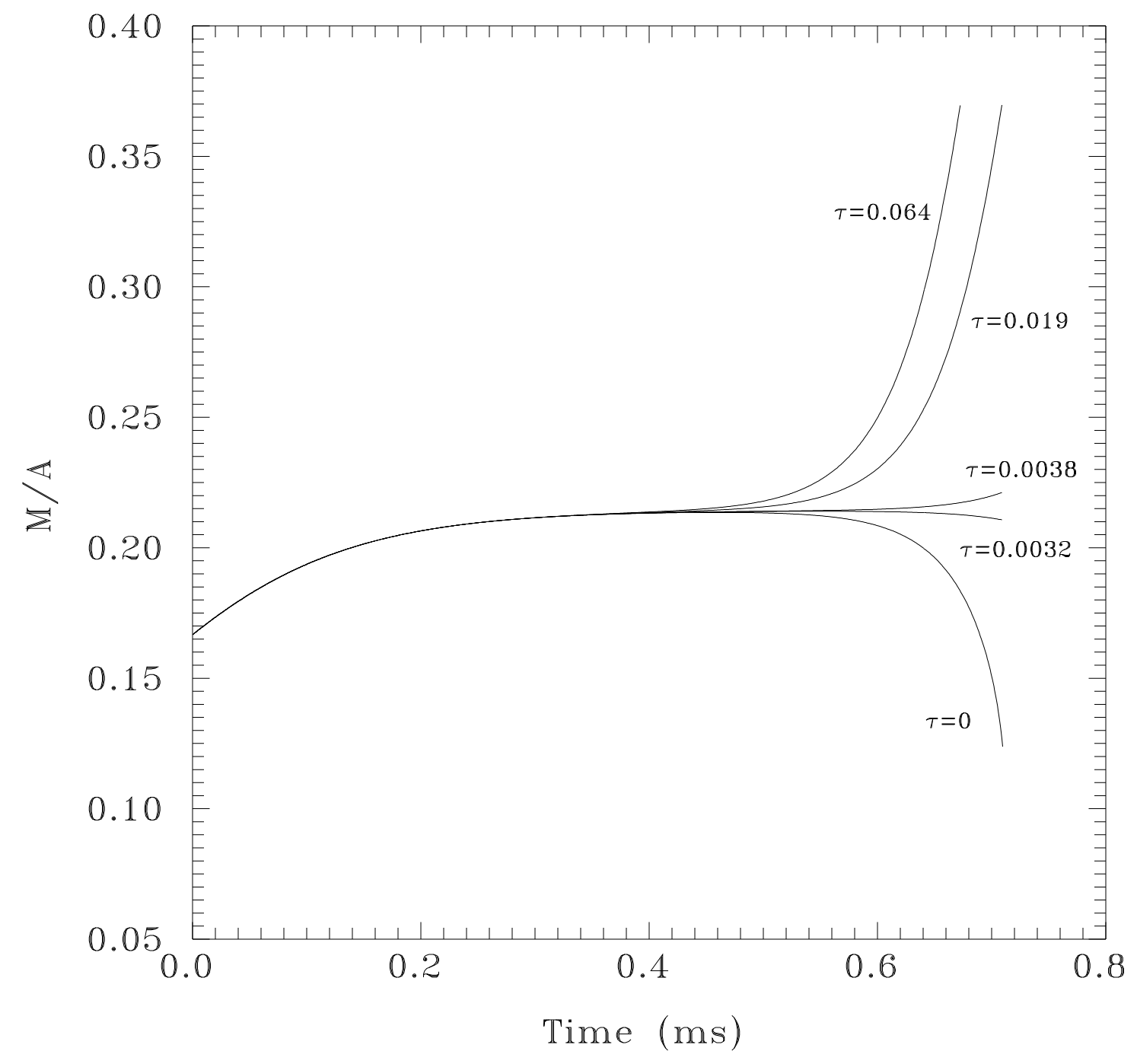




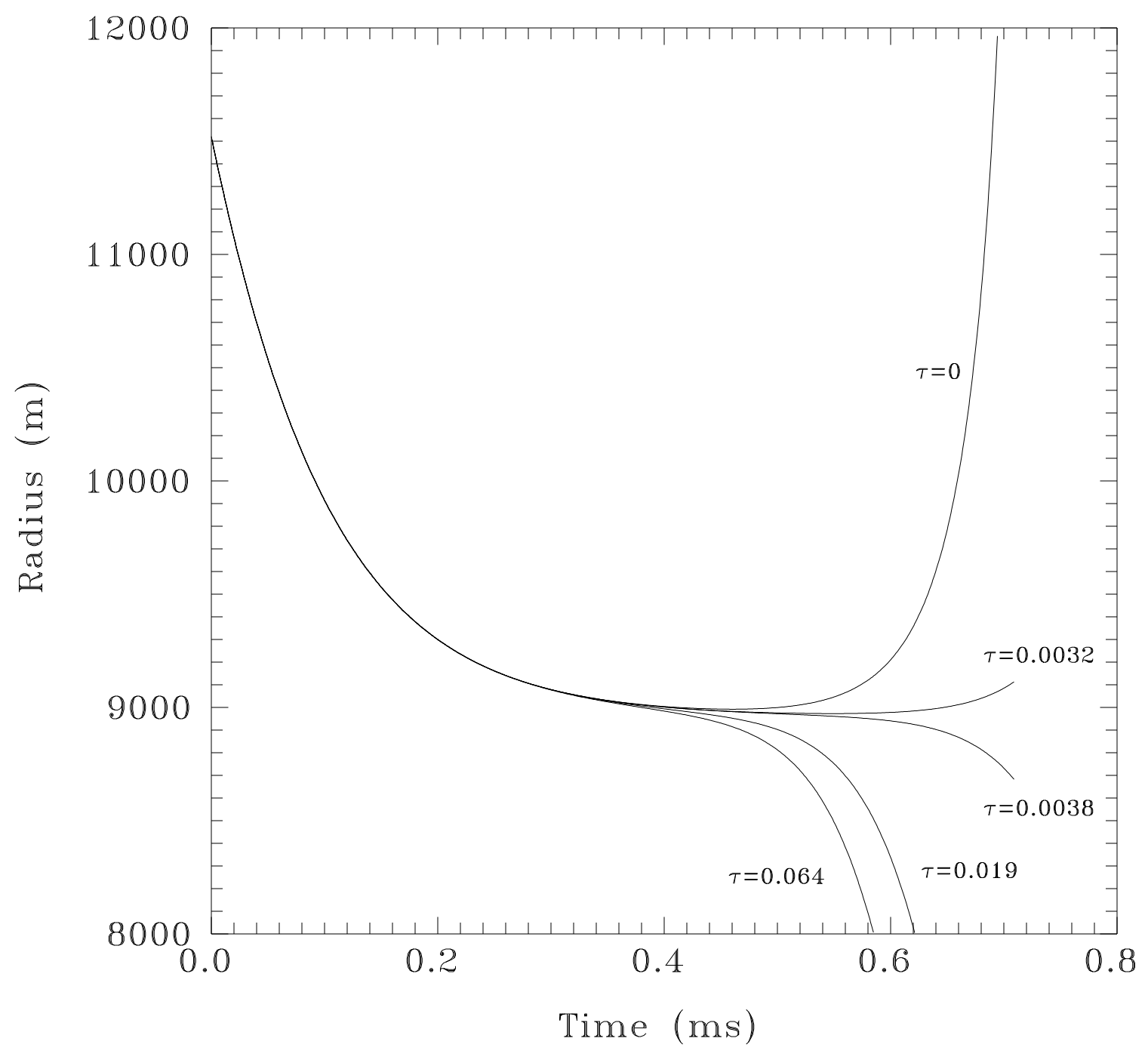




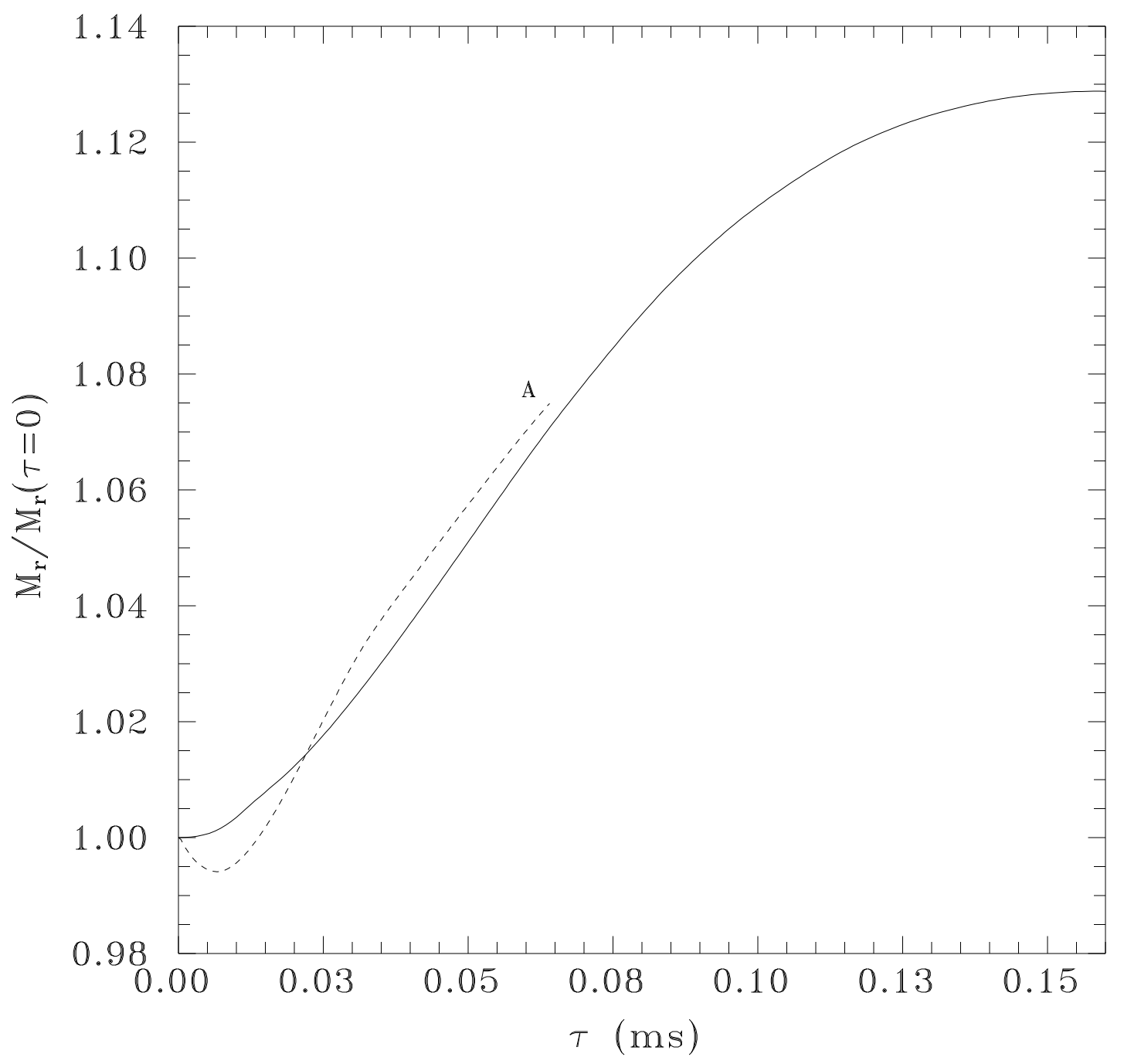




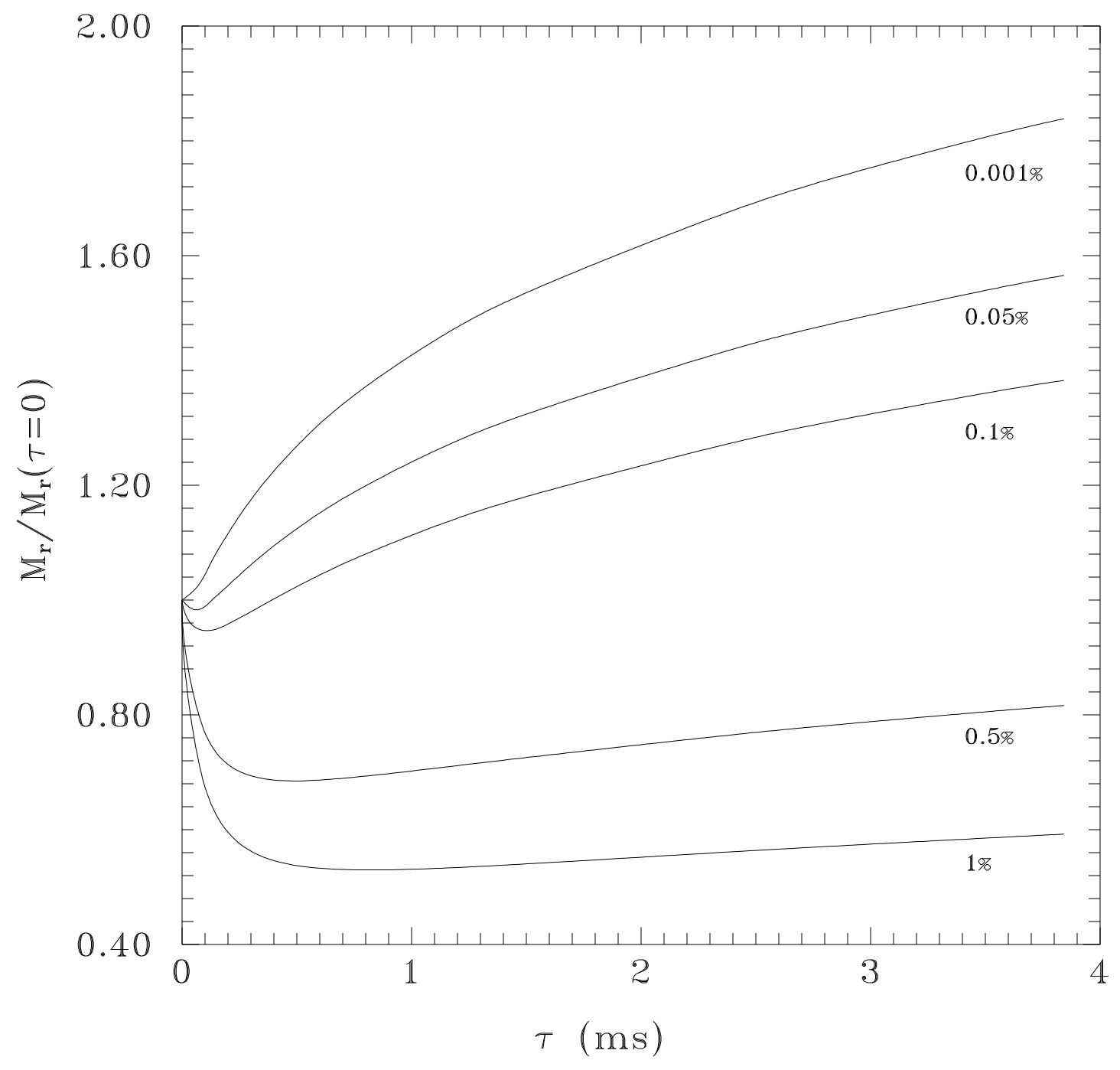




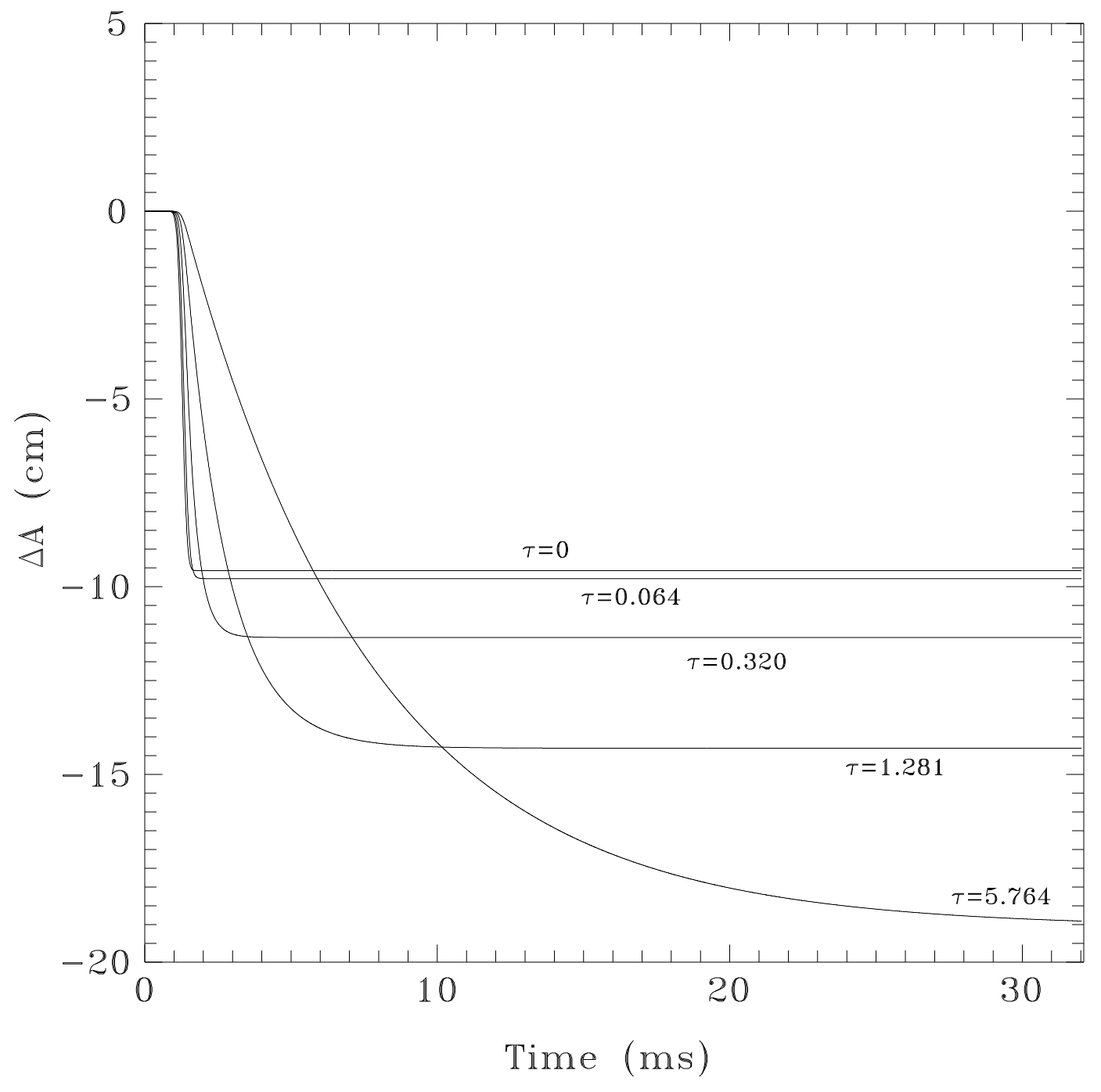




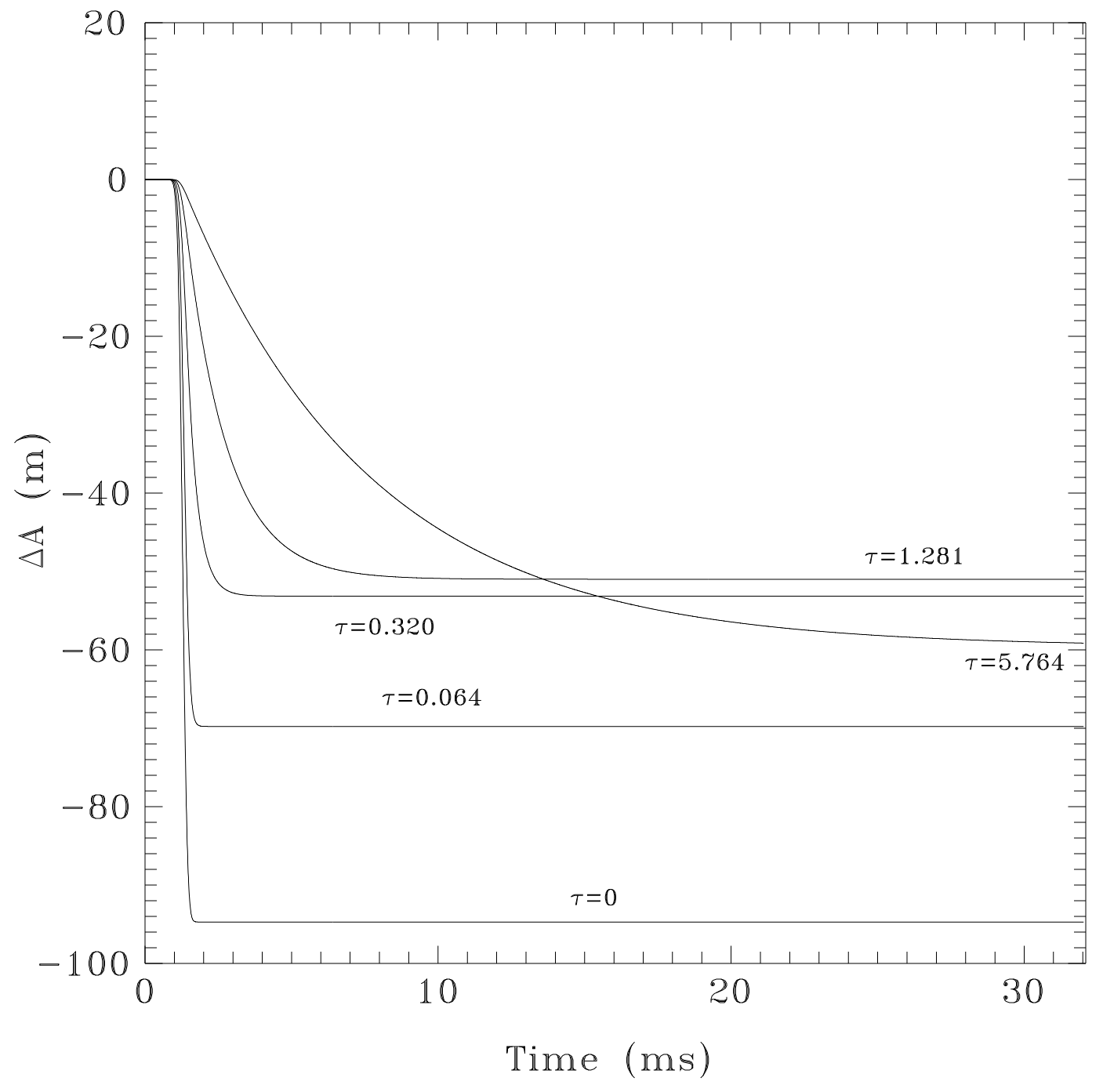




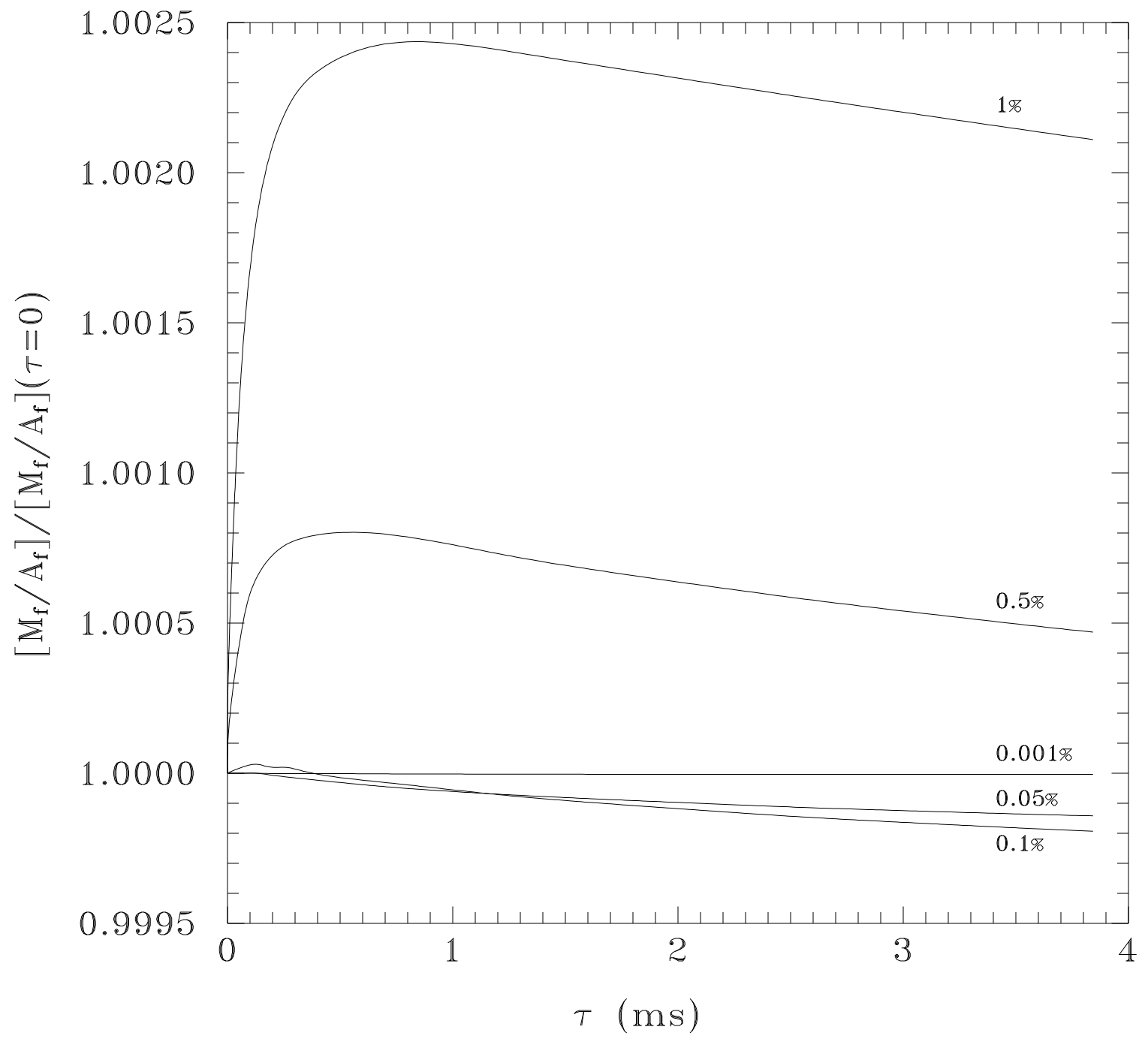




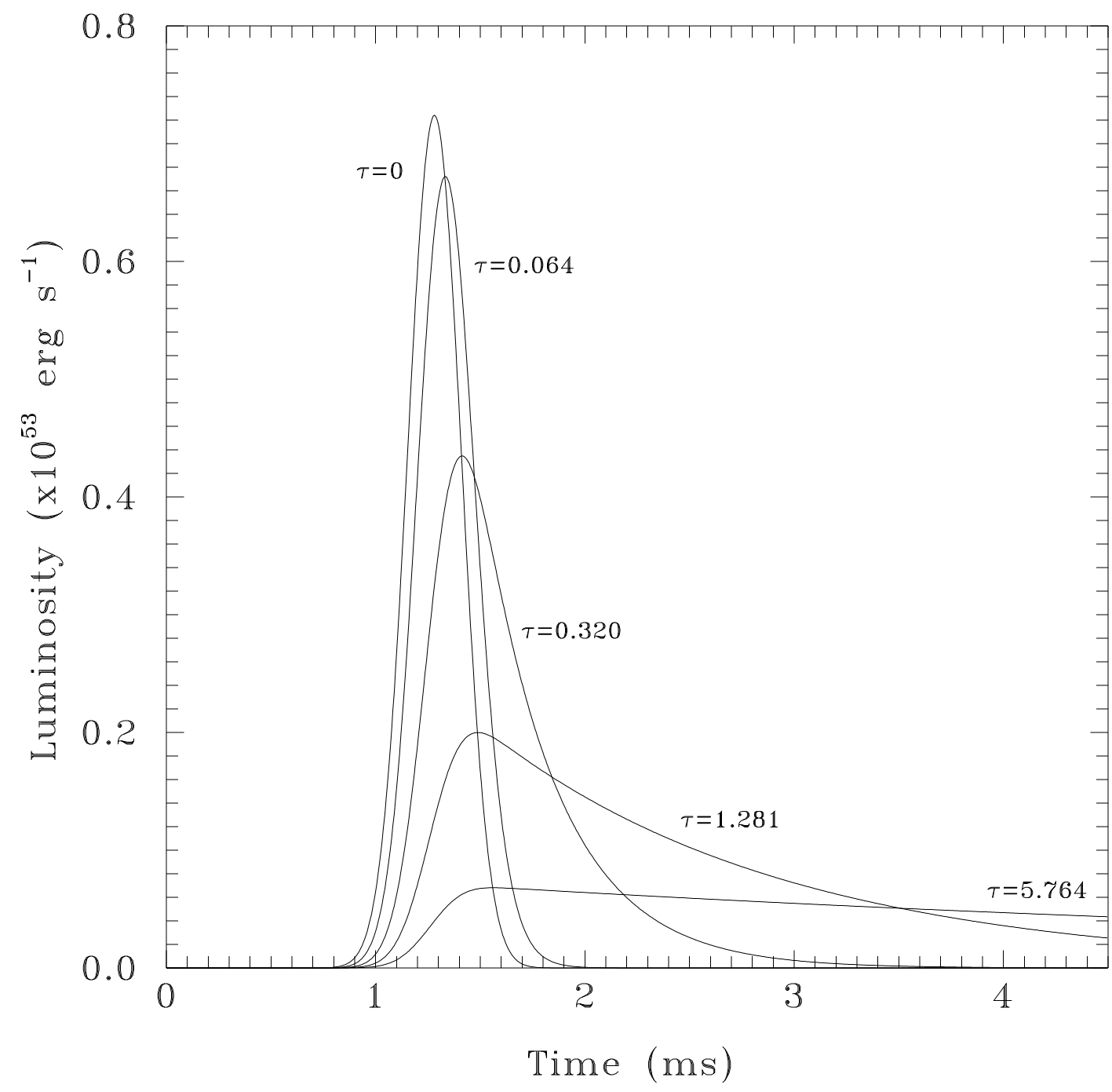




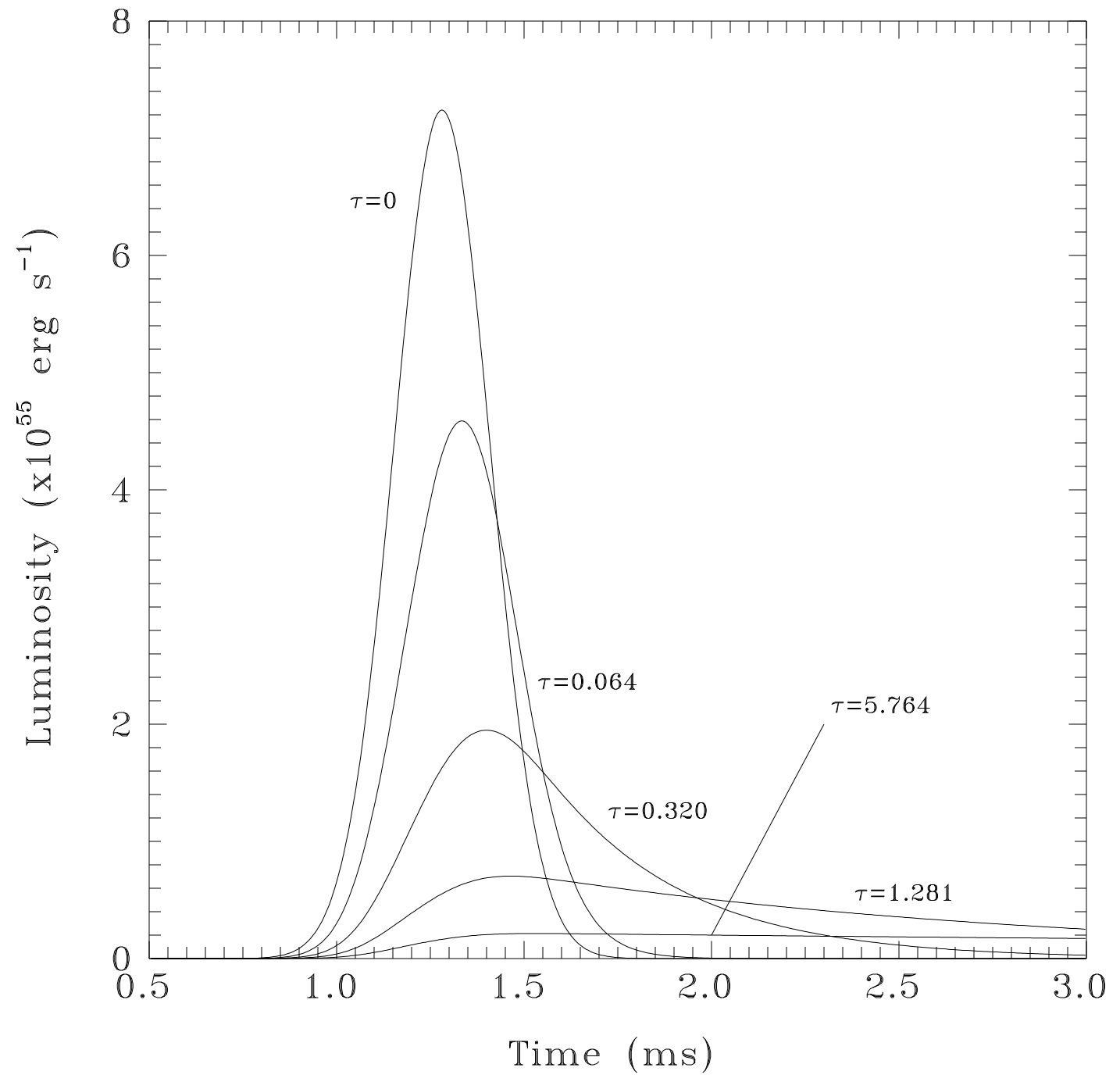

